\title{
Directional reversals and multimode dynamics in semiconductor ring lasers
}

\author{
Antonio Pérez-Serrano* \\ Weierstrass Institute for Applied Analysis and Stochastics, WIAS, Berlin, D-10117, Germany \\ Julien Javaloyes ${ }^{\dagger}$ \\ Departament de Física, Universitat de les Illes Balears, UIB, Palma de Mallorca, E-07122, Spain \\ Salvador Balle $\mathrm{e}^{\ddagger}$ \\ Institut Mediterrani d'Estudis Avançats, IMEDEA, UIB-CSIC, Esporles, E-07190, Spain
}

(Received 2 December 2013; published 14 February 2014)

\begin{abstract}
We investigate the dynamics of longitudinal modes in quantum-well semiconductor ring lasers by means of a spatiotemporal traveling-wave model. We report the existence of a multimode instability in such a system that provokes a periodic deterministic directional reversal involving jumps between consecutive longitudinal modes. The switching sequence follows the modal frequencies from blue to red, and every modal jump is accompanied by a reversal of the direction of emission. We characterize and analyze this instability via a bifurcation analysis of the full traveling-wave model as well as by performing a linear stability analysis of the monochromatic solutions.

DOI: 10.1103/PhysRevA.89.023818

PACS number(s): 42.55.Px, 42.60.Mi, 42.65.Pc, 42.65.Sf
\end{abstract}

\section{INTRODUCTION}

Semiconductor ring lasers (SRLs) are interesting and promising laser sources from diverse perspectives. From the technological point of view, they do not require cleaved facets to form a resonant cavity, they can be tested on a wafer scale before dicing, and they can emit in a single mode without the use of Bragg gratings. Therefore they are simpler to fabricate and to integrate in photonic integrated circuits [1] than either Fabry-Pérot (FP) or distributed feedback lasers. From the physical point of view, SRLs are very interesting devices because they show a rich variety of dynamical behaviors including bidirectional continuous-wave (Bi-CW) operation [2], intensity alternate oscillations (AOs) between counterpropagating electric fields [3], unidirectional (UNI) bistable emission [4], directional multistability [5], and cavityenhanced four-wave mixing (FWM) [6].

At relatively high pump currents the strong competition for the gain imposes that only one of the two counterpropagating fields can be active, thereby leading to directional bistability. In this case the direction of emission can be switched by optical trigger pulses at ultrafast speeds $[7,8]$. Directional bistability has been extensively investigated due to its direct application for performing all-optical processing, because unlike previously proposed optical bistables [9], SRLs meet simultaneously all the stringent requirements for the development of viable integrated functional photonics, such as speed, small footprint, low switching energy, and easy read-write mechanism. This feature has been exploited to demonstrate applications such as all-optical memories [10], logic gates [11], flip-flops [12], random bit generators [13,14], and data processors [15].

In addition, the emission wavelength of a SRL exhibits multistability and can be switched either by optical trigger

\footnotetext{
*Antonio.Perez-Serrano@wias-berlin.de

†julien.javaloyes@uib.es

†salvador@imedea.uib-csic.es
}

pulses [16] or by wavelength-selective feedback [12,17]. The coexistence and nonlinear interaction between directional and spectral domain modes provides rich opportunities for implementing basic logical functionalities, e.g., a two-bit memory was demonstrated using wavelength and directional multistability between two cavity modes [18].

This variety of dynamical behaviors has been successfully described by means of a traveling-wave model (TWM) that incorporates a mesoscopic approximation to the optical response of semiconductor quantum-well $(\mathrm{QW})$ media valid for time scales longer than 1 ps [19-21]. Direct integration of the TWM has allowed successful explanation of the main dynamical characteristics of free-running SRLs, including lasing direction hysteresis [22], and highlighting of the strong impact that residual reflections in the light extraction sections have on the selection of the lasing mode [23]. The TWM has also been used to investigate directional switching [24] and FWM in SRLs subject to optical injection [25].

In this paper, we present and analyze a multimode instability in SRLs that consists of a periodic reversal-on a slow time scale of tens of nanoseconds-of the direction of emission that occurs through jumps between consecutive longitudinal modes. Between reversals, the SRL operates in a single-mode UNI regime, and at each jump the frequency of emission proceeds from blue to red. Although it bears some similarity with the instability reported in $[26,27]$ for FP lasers, where the emission of the laser passed from the bluest longitudinal mode to the reddest, in our case every modal jump is accompanied by a directional reversal which has important implications. We show that due to the extra degree of freedom that stems from the directional bistability, the threshold for this instability is much lower than it is in FP lasers, which can hinder the performance of SRLs at relatively low bias current. Our theoretical analysis is based on a linear stability analysis (LSA) of the monochromatic solutions of the full spatiotemporal TWM for semiconductor media, which generalizes the method we developed in the past for the simpler two-level atom laser [28]. Our results reveal that, while the mechanism 
that produces the modal jumps from the bluest toward the reddest frequencies is the same in both FP and SRLs - the asymmetry of the gain spectrum [26,27], the $\alpha$ factor, and the so-called Bogatov effect [29] — the directional reversals are energetically preferred in unidirectional SRLs because they allow FWM effects to be avoided and involve mixing only between two detuned counterpropagating waves. This result is particularly surprising since the population grating involved in the directional reversal consists of a half-wavelength grating that is strongly washed out by carrier diffusion.

The paper is organized as follows. In Sec. II, the theoretical methods used are described, including a summary of the TWM and the methods used to perform its numerical integration and bifurcation analysis. Further technical details on the numerical implementation of the LSA for the TWM in the general case are given in Appendix A. In Sec. III we present the multimode instability obtained by numerical integration of the TWM. In order to explain its dynamical origin, an approximate LSA is performed in the uniform field limit (UFL) [30] (see Appendix B for the details), which is compared to the numerical LSA in the general case.

\section{THEORETICAL METHODS}

Our analysis of the multilongitudinal mode dynamics in SRLs is based on the direct integration of a TWM [22] as well as on the LSA of its monochromatic solutions, which allow us to perform their bifurcation analysis. In this section we briefly summarize these theoretical tools.

\section{A. Traveling-wave model}

We summarize here the TWM developed in [22] for the slowly varying amplitudes of the clockwise and counterclockwise fields $E_{ \pm}(z, t)$ of a quasimonochromatic field around an optical carrier frequency $\omega_{0}$ and carrier propagation constant $q_{0}=n_{0} \omega_{0} / c$, where $n_{0}$ is the effective index of the waveguide for the TE mode. The presence of the counterpropagating fields imposes on the carrier density $N(z, t)$ a spatial modulation at half the optical wavelength; hence we take

$$
N(z, t)=N_{0}(z, t)+N_{+2}(z, t) e^{2 i q_{0} z}+N_{-2}(z, t) e^{-2 i q_{0} z},
$$

where $N_{0}(z, t)$ is the local average of the carrier density and $N_{+2}(z, t)=N_{-2}^{*}(z, t)$ describes the amplitude of the carrier spatial modulation at half the optical wavelength. The grating variables $N_{ \pm 2}(z, t)$ describe the so-called short-range spatial hole burning, which is due to the standing-wave character of the field and the associated variation of the carrier density on the spatial scale of the emission wavelength. Although small, this effect can have a strong influence on the field dynamics, and it is the principal ingredient of the transition from Bi-CW toward bistable UNI emission in SRLs. It is also capable of provoking a synchronization transition [31,32], although this effect is more easily seen in cold atom vapors [33] where diffusive effects are mitigated. On the other hand, long-range SHB consists of the spatial variation of the carrier density $N_{0}(z, t)$ and it is mainly due to the departure from conservative behavior and from the UFL limit of either the point coupler reflection and transmission coefficients in a SRL or the mirror reflectivities in FP lasers.
Scaling space and time to the ring length $L_{r}$ and the ring transit time $\tau_{r}=n_{g} L_{r} / c$, where $n_{g}$ is the effective group index, our TWM reads

$$
\begin{gathered}
\left(\partial_{t} \pm \partial_{z}\right) E_{ \pm}=i P_{ \pm}-\alpha_{i} E_{ \pm}, \\
\partial_{t} N_{0}=J-R\left(N_{0}\right)-i\left(P_{+} E_{+}^{*}+P_{-} E_{-}^{*}-\text { c.c. }\right), \\
\partial_{t} N_{ \pm 2}=-\left[R^{\prime}\left(N_{0}\right)+\eta\right] N_{ \pm 2}-i\left(P_{ \pm} E_{\mp}^{*}-E_{ \pm} P_{\mp}^{*}\right),
\end{gathered}
$$

where $2 \alpha_{i}$ are the internal losses, $J$ is the injected current density, $R(N)=A N+B N^{2}+C N^{3}$ describes carrier recombination, which is modeled with a cubic fitting, $R^{\prime}=d R / d N$ is the effective interband carrier relaxation rate, and $\eta=4 \mathcal{D} q_{0}^{2}$ where $\mathcal{D}$ is the ambipolar diffusion coefficient. In this model, carrier diffusion is included in the evolution of the grating terms $N_{ \pm 2}(z, t)$ only; its effect is negligible on $N_{0}(z, t)$ because the characteristic scale of $N_{0}(z, t)$ is the cavity length.

The slowly varying amplitudes of the polarizations of the QW medium, $P_{ \pm}(z, t)$, are determined from a mesoscopic approximation to the optical response of the semiconductor QW material [19] and computed via a convolution integral [21],

$$
\begin{aligned}
P_{ \pm}(z, t)= & \int_{0}^{\infty} d t^{\prime}\left\{\chi\left[t^{\prime}, N_{0}(z, r)\right] E_{ \pm}(z, r)\right. \\
& \left.+\chi_{N}\left[t^{\prime}, N_{0}(z, r)\right] N_{ \pm 2}(z, r) E_{\mp}(z, r)\right\}+\beta \xi_{ \pm}(z, t),
\end{aligned}
$$

where $r=t-t^{\prime}$. The convolution kernel has the form

$$
\chi\left(t^{\prime}, N\right)=\chi_{0} e^{-\left[\gamma+i\left(\Omega_{G}-\omega_{0}\right)\right] t^{\prime}} \frac{2 e^{-i \gamma N t^{\prime}}-1-e^{-i \Omega_{T} t^{\prime}}}{t^{\prime}},
$$

where $\gamma$ is the polarization decay rate, $\Omega_{G}$ is the photon frequency corresponding to the gap, and $\Omega_{T}$ is the maximum photon frequency absorbed by the QW medium. Also, $\chi_{N}=$ $\partial \chi / \partial N$ denotes the variation of $\chi\left(t^{\prime}, N\right)$ with carrier density. For the sake of simplicity we use the convolution kernel (5) instead of the one developed in [34] where the electron-hole tails and the effect of the temperature are included. We also add spontaneous emission of the amplitude $\beta$ by including a Gaussian white noise term $\xi_{ \pm}(z, t)$ of zero mean and correlation $\left\langle\xi_{ \pm}(z, t) \xi_{ \pm}(\hat{z}, \hat{t})\right\rangle=\delta(t-\hat{t}) \delta(z-\hat{z})$.

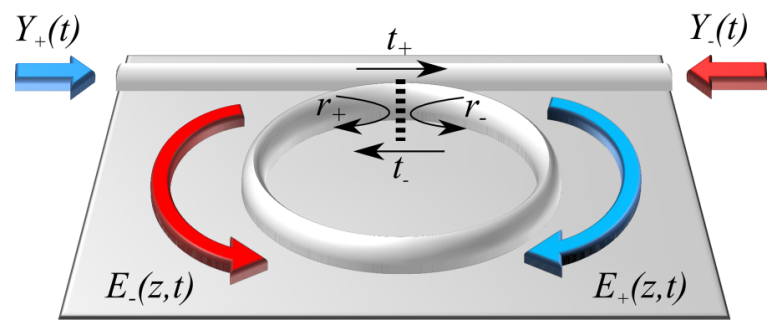

FIG. 1. (Color online) Representation of the semiconductor ring laser. For the sake of simplicity in our model the input-output waveguide is supposed to be transparent and the coupling with the ring cavity described as one point (dashed black line) that imposes the boundary conditions described in Eqs. (6) where $t_{ \pm}$and $r_{ \pm}$are the transmission and reflection coefficients for the counterpropagating fields $E_{+}(z, t)$ and $E_{-}(z, t) . Y_{ \pm}(t)$ are the injected fields for each direction. 
The TWM defined by Eqs. (1)-(4) has to be closed with the boundary conditions for the ring cavity (see Fig. 1). For the sake of simplicity we consider an optical carrier frequency that corresponds to a cavity mode and we do not take into account the length and the frequency dependence of the light extraction sections [23]; hence the boundary conditions read

$$
\begin{aligned}
& E_{+}(0, t)=t_{+} E_{+}(1, t)+r_{-} E_{-}(0, t)+Y_{+}(t), \\
& E_{-}(1, t)=t_{-} E_{-}(0, t)+r_{+} E_{+}(1, t)+Y_{-}(t),
\end{aligned}
$$

where $t_{ \pm}$and $r_{ \pm}$are the transmission and reflection coefficients at the output coupler for the $E_{ \pm}$fields, respectively. $Y_{ \pm}(t)$ are the external fields injected in each propagation direction, respectively, which we assume to be Gaussian pulses of the form

$$
Y_{ \pm}(t)=\sqrt{I_{ \pm}} \exp \left(\frac{-t^{2}}{4 \sigma_{ \pm}^{2}}-i \omega_{ \pm} t\right)
$$

hence their pulse energy is $E_{p}^{ \pm}=I_{ \pm} \sigma_{ \pm} \sqrt{2 \pi}$ and their pulse FWHM $\tau_{ \pm} \simeq 2.355 \sigma_{ \pm}$.

\section{B. Numerical integration}

Numerical integration and analysis of the TWM is performed after recasting it into an ensemble of delayed algebraic equations (DAEs) [35] using a spatial discretization of $N=$ 401 points (corresponding to a time step $\Delta t=31.2 \mathrm{fs}$ ) with a decimation factor $D=25$; the convolution kernels are computed using $M=26$ points in the past. The numerical algorithm used to integrate Eqs. (1)-(4) can be found in [36,37]. In addition to obtaining shorter simulation times, recasting the TWM into DAEs causes the degrees of freedom of the system to decrease considerably, allowing us to perform the LSA of the system of Eqs. (1)-(4) in a similar way as we did in [28] and summarized in Sec. II C. Unless otherwise indicated, the

\begin{tabular}{|c|c|c|c|}
\hline Symbol & Value & Units & Meaning \\
\hline \multicolumn{4}{|c|}{ Waveguide parameters } \\
\hline$\lambda_{0}$ & 1550 & $\mathrm{~nm}$ & Emission wavelength \\
\hline$n_{g}$ & 3.6 & & Effective group index \\
\hline$\tau_{r}$ & 12.5 & ps & Ring transit time \\
\hline$L_{r}$ & 1.04 & $\mathrm{~mm}$ & Length of the ring cavity \\
\hline $2 \alpha_{i}$ & 14.4 & $\mathrm{~cm}^{-1}$ & Internal losses \\
\hline$t_{ \pm}$ & 0.95 & & Transmission coefficients \\
\hline$r_{ \pm}$ & $(10+i 5) \times 10^{-5}$ & & Reflection coefficients \\
\hline \multicolumn{4}{|c|}{ Active material parameters } \\
\hline$N_{t}$ & $1 \times 10^{18}$ & $\mathrm{~cm}^{-3}$ & Transparency carrier density \\
\hline $\mathcal{D}$ & 11.6 & $\mathrm{~cm}^{2} \mathrm{~s}^{-1}$ & Ambipolar diffusion coefficient \\
\hline$A$ & $1 \times 10^{-8}$ & $\mathrm{~s}^{-1}$ & Recombination coefficient \\
\hline$B$ & $7 \times 10^{-10}$ & $\mathrm{~cm}^{3} \mathrm{~s}^{-1}$ & Recombination coefficient \\
\hline$C$ & $1 \times 10^{-29}$ & $\mathrm{~cm}^{6} \mathrm{~s}^{-1}$ & Recombination coefficient \\
\hline $2 \chi_{0}$ & 72 & $\mathrm{~cm}^{-1}$ & Maximum modal gain \\
\hline$\gamma$ & $8 \times 10^{12}$ & $\operatorname{rad~s}^{-1}$ & Polarization decay rate \\
\hline$\Omega_{T}$ & $9 \times 10^{13}$ & $\operatorname{rad~s}^{-1}$ & Top of the band frequency \\
\hline$\Omega_{G}$ & $5 \times 10^{12}$ & $\operatorname{rad~s}^{-1}$ & Band-gap frequency \\
\hline$\beta$ & $1 \times 10^{-4}$ & & Spontaneous emission \\
\hline
\end{tabular}
parameters used are shown in Table I.

TABLE I. Model parameters.

\section{Bifurcation analysis}

The bifurcation analysis of the TWM is a particularly demanding task because the system of partial differential equations that defines the TWM is hyperbolic, thereby presenting advection. As a consequence, it cannot be recast into an ensemble of sparsely coupled ordinary differential equations (ODEs) by the method of lines [38] which allows the use of software packages like AUTO [39] or DDE-BIFTOOL [40] for performing the numerical bifurcation analysis of ODEs and delay differential equations (DDEs). Our method, summarized here and detailed in Appendix A, is based on the discretized temporal map that advances the solution in time while verifying the Courant-Friedrich-Levy (CFL) condition [41]. It allows us to map the different regimes encountered when varying one or several parameters, thereby providing us with the global dynamical scenario.

This is a two-step process: It requires in the first place finding the monochromatic solutions of the system, and then performing their LSA as one control parameter is scanned. In our past work [28], the simplicity of the two-level atom description allowed us to find the monochromatic solutions of the system by using a shooting method. However, this procedure cannot be directly applied to the present case due to the nonlinear dependence of the convolution kernel on the carrier density, which defines a highly multidimensional nonlinear problem that can be difficult to solve unless a good guess solution is provided.

\section{Monochromatic solutions}

Numerically, the monochromatic solutions are represented as a state vector $\vec{V}$ formed by the $\mathcal{N}$ (real) values needed to specify all the variables at each spatial point, including past values as required to describe both propagation over the decimated mesh and the convolution kernels that yield the polarizations. In order to find these monochromatic solutions, we start from the trivial off solution and we perform its LSA as described below for different values of the control parameter to be scanned, for instance, the current density $J$. For each value of the parameter, we find the eigenvalues that cross the imaginary axis-if any-and their associated eigenvectors. This parameter value represents the threshold of a lasing branch, and we use the eigenvector as the guess solution to solve the multidimensional nonlinear problem for $\vec{V}$ using a Newton-Raphson algorithm that converges after a few iterations to a bidirectional solution on the corresponding lasing branch. After the different lasing branches have been determined, it is easy to continue each of them by changing the parameter and solving the multidimensional problem using the previous solution as a guess. After that, the LSA is performed to determine the stability of each branch solution as a function of the parameter.

\section{Linear stability analysis}

The method used to perform the LSA of the monochromatic solutions of the TWM (1)-(4) is based on the fact that the evolution of any state vector $\vec{V}(t)$ over a time step $h$ can be written as a temporal map $\vec{V}(t+h)=\vec{U}(h, \vec{V}(t))$, where $\vec{U}(h, \vec{V})$ verifies the CFL condition and cancels numerical dissipation [41]. Considering the perturbations $\vec{v}$ around a 
monochromatic solution $\vec{V}(t)$, one finds the matrix $\mathbf{M}=$ $\partial \vec{U} / \partial \vec{V}$ representing the linear operator that governs the time evolution of the perturbations around $\vec{V}(t)$. One finally computes the $\mathcal{N}$ Floquet multipliers $z_{\mathcal{N}}$ of $\mathbf{M}$ via a $\mathrm{QR}$ decomposition method [42]; these determine the eigenvalues as $\lambda_{\mathcal{N}}=h^{-1} \ln z_{\mathcal{N}}$. As usual, if none of these computed eigenvalues has a positive real part, then one concludes that this monochromatic solution is stable, and unstable otherwise. The details of the numerical implementation of the LSA can be found in Appendix A. Please notice that the method also allows finding the associated eigenvectors, which will prove important in determining the most unstable directions in phase space.

\section{RESULTS AND DISCUSSION}

\section{A. Modal multistability}

For the parameters in Table I, the SRL shows longitudinal mode bistability between modes $m=0(f=0)$ and $m=-1$ $(f=-78 \mathrm{GHz})$ when it is biased in the UNI regime, for $J>2$. In this situation, and in agreement with experimental results $[16,18]$, the emission direction and frequency can be switched by optical trigger pulses. Figure 2 shows how the switching from mode $m=0$ to mode $m=-1$ at $J=2.5$ is accomplished by injection of a Gaussian pulse as described in Eq. (7) with $I_{ \pm}=3, \tau_{ \pm}=100 \mathrm{ps,} \mathrm{and} \mathrm{spectrally} \mathrm{centered}$ over mode $m=-1$ in the copropagating (left column) and counterpropagating (right column) directions with respect to the previous steady state, a clockwise state at $m=0$. In both cases, the modal intensities of $m=0$ and $m=-1$ behave in the same way: Mode $m=0$ drops to a very low value almost instantaneously, while mode $m=-1$ switches on through damped relaxation oscillations. The main difference between the two cases is that for the copropagating case, the beating of the modal amplitudes in the total intensity is much more evident than for the counterpropagating case, which already indicates that switching to a copropagating mode implies a transient involving more longitudinal modes than when switching to a counterpropagating mode.
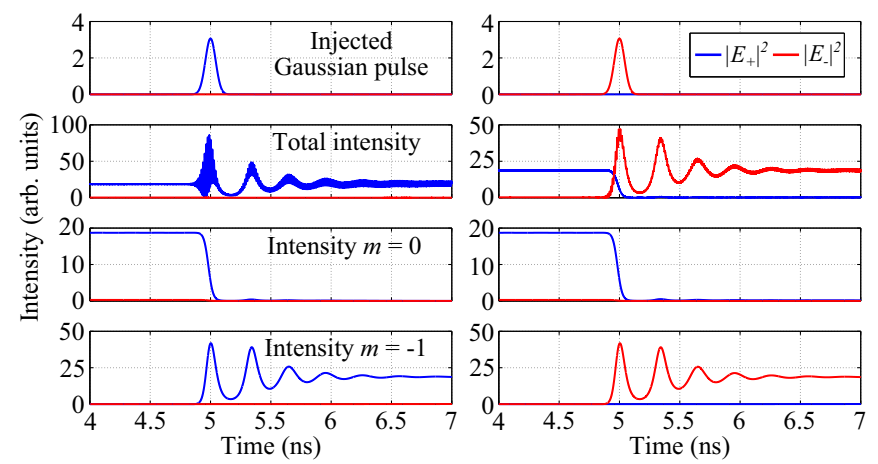

FIG. 2. (Color online) Switching upon injection of a copropagating (left column) and a counterpropagating (right column) Gaussian pulse with $I_{ \pm}=3, \tau_{ \pm}=100 \mathrm{ps}$, and $m=-1(f=-78 \mathrm{GHz})$. From top to bottom, the panels display the Gaussian pulse intensity, the total intensity, and the modal intensities for modes $m=0$ and $m=-1$, obtained by filtering the total intensity around each mode. The initial state was at $m=0(\omega=0) . J=2.5$.

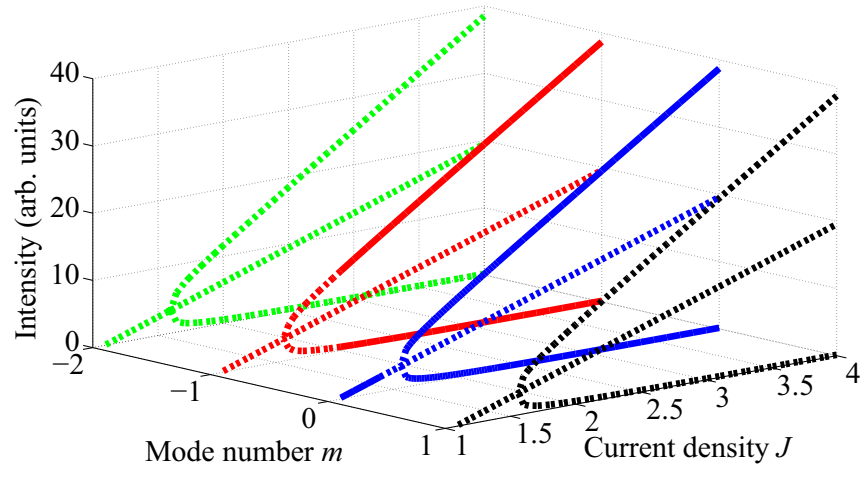

FIG. 3. (Color online) Bifurcation diagrams of the dominant modes of a SRL with the parameters in Table I. The solid (dashed) lines indicate stable (unstable) monochromatic solutions. Modes $m=0$ and $m=-1$ share a current region where they are stable in the UNI regime; thus there is longitudinal mode bistability. The other modes are unstable for the explored current density values.

Such a situation is encountered over a large current range, and indeed is well reproduced by the LSA of the different monochromatic solutions. The bifurcation diagram for the dominant modes of a SRL with the parameters in Table I is shown in Fig. 3. It is seen that mode $m=0$ is selected at threshold $\left(J_{t h} \simeq 1.1\right)$ and that it starts to stably lase bidirectionally (Bi-CW) up to $J \simeq 1.4$, where a Hopf bifurcation occurs (see Fig. 13 for details) that leads to the AO regime. Finally, a symmetry-breaking pitchfork bifurcation takes place after the AO Hopf bifurcation on the Bi-CW solution. This branch eventually collides with the AO limit cycle as described in [5]; this collision changes the stability of the branch and leads to the unidirectional bistable regime (UNI) for currents $J \geqslant 1.65$. The mode $m=-1$ presents similar characteristics, but stable lasing on this mode is possible only for currents $J>2$. Above this value of $J$, both modes can operate stably in the UNI regime, thus leading to a region of multistability of longitudinal modes where the SRL can emit at different wavelengths and in different propagation directions. On the other hand, the other modes are all unstable for the range of pump values explored.

Interestingly, the range of longitudinal mode multistability is asymmetric with respect to mode $m=0$ in spite of having mode $m=0$ exactly at the peak gain. This is clearly different from the two-level atom case, where the range of multistability was symmetric around the gain peak for mode $m=0$ at the gain peak [28]. This is due to the amplitude-phase coupling that occurs in semiconductor materials, which leads to an asymmetry of the gain curve, and the associated Bogatov effect, which implies an asymmetric saturation of the gain [29]. In our case, $\alpha$ is not an input parameter, but it arises through the complex response function of the material; as such, it depends on both operation frequency and carrier density, and in the present case it is $\alpha \simeq 1.16$.

\section{B. Switching instability}

For the parameters in Table I, only two modes are stable up to $J=4$, but the number of stable modes strongly depends on the parameter values, in particular, on the mode spacing as compared to the width of the gain spectrum. Moreover, the 


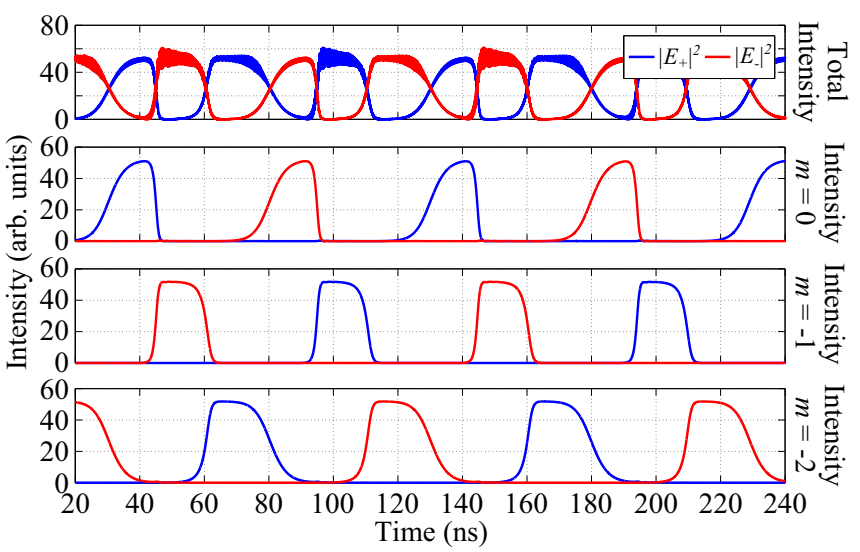

FIG. 4. (Color online) Longitudinal mode switching instability at $J=5$. The period of the oscillation is $100 \mathrm{~ns}$. Parameters as in Table I except $\mathcal{D}=2.32 \mathrm{~cm}^{2} \mathrm{~s}^{-1}$.

carrier diffusion $\mathcal{D}$ plays an important role in the longitudinal mode multistability: it was shown in [28] that FP lasers can exhibit multistability if diffusion is strong enough to wash out the carrier grating, while in SRLs in the UNI regime the carrier grating is always small. Hence, one could expect that multistability in SRLs will disappear for a stronger carrier grating; this can be accomplished either by reducing carrier diffusion or by working at longer wavelengths (i.e., telecom wavelengths), since both effects reduce $\eta$ in Eq. (3). In fact, for a diffusion coefficient $\mathcal{D}=2.32 \mathrm{~cm}^{2} \mathrm{~s}^{-1}$ and the other parameters as in Table I, the output of the SRL becomes unstable (see Fig. 4), with the direction of emission switching back and forth periodically at a low frequency of a few megahertz. Moreover, several modes are involved in the dynamics, but at any given time the laser emits essentially on a single longitudinal mode. Thus this instability does not arise from the locking of different modes giving pulsed operation at the cavity round-trip time like the Risken-Nummedal-GrahamHaken instability [43], and it is also different from the unstable behavior due to mode competition reported in [44]. Instead, the multimode instability shown in Fig. 4 is similar to the one reported in [26] for FP lasers: there are periodic intensity fluctuations of each mode and the switching sequence follows the modal frequencies from blue to red; when the reddest mode switches off, the sequence restarts from the bluest mode. However, in our case each switching has associated a change in the emission direction.

\section{Analysis of the switching instability}

The first aspect to remark is that the switching instability exists only in a limited current range. For the parameters considered here, when the current is too high $(J>6)$ the switching becomes incomplete and unsteady multimode emission is obtained. As an example, Fig. 5 shows the output of the SRL for $J=6.5$, which evidences that the emission is mainly in the clockwise direction, although periodic bursts of light in the counterclockwise direction also occur. Interestingly, the emission in the clockwise direction is dominated by mode $m=-2$, while that in the counterclockwise direction is dominated by mode $m=0$, although in both directions the

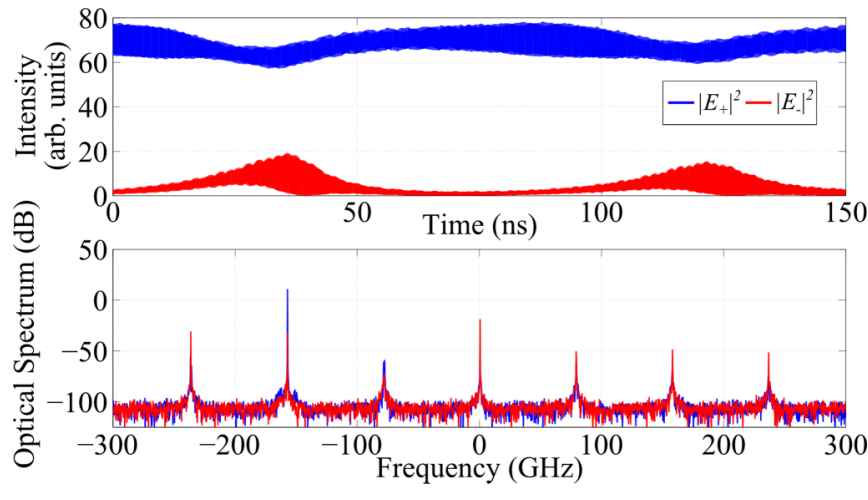

FIG. 5. (Color online) Time trace for $J=6.5$ (upper panel) and the optical spectrum (lower panel). Parameters as in Table I except $\mathcal{D}=2.32 \mathrm{~cm}^{2} \mathrm{~s}^{-1}$.

emission is no longer almost single mode, thus leading to the fast beat note visible in the direction-resolved traces.

Within the range of existence of the instability, the residence time on the different modes involved in the cycle-hence the period - strongly depends on the current density $J$, as shown in Fig. 6. When $J$ is increased, the residence time for mode $m=-1$ monotonically decreases from infinity (when the mode is stable) toward a finite, small value. At the same time, the residence time on modes $m=0$ and $m=-2$ increases monotonically from zero (when mode $m=-1$ is stable) until they dominate the dynamics and the instability is destroyed (see Fig. 5). In this range, the period of the cycle has a minimum at $J \simeq 5$, when the residence times on the three modes involved in the cycle are the same.

A deeper understanding of the switching dynamics can be gained by performing a bifurcation analysis of the monochromatic solutions of the system; see Fig. 7. It can be seen that $m=0$ starts to lase in the Bi-CW regime but only for a small current range; then a pitchfork bifurcation leads to the UNI regime. In the UNI regime, mode $m=0$ eventually becomes unstable at $J \simeq 2$. On the other hand, UNI solutions on mode $m=-1$ become stable at $J \simeq 1.5$-thus leading to a small region of directional and wavelength bistability with mode $m=0$ - and so remain up to $J \simeq 4$. Above this point, $J>4$,

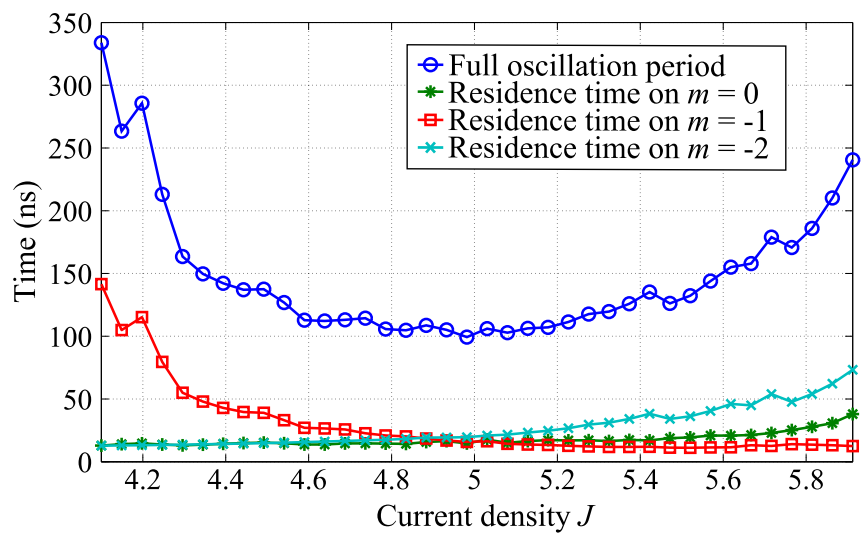

FIG. 6. (Color online) Time characterization of the longitudinal mode switching instability shown in Fig. 4 for different current density $J$ values. Parameters as in Table I except $\mathcal{D}=2.32 \mathrm{~cm}^{2} \mathrm{~s}^{-1}$. 


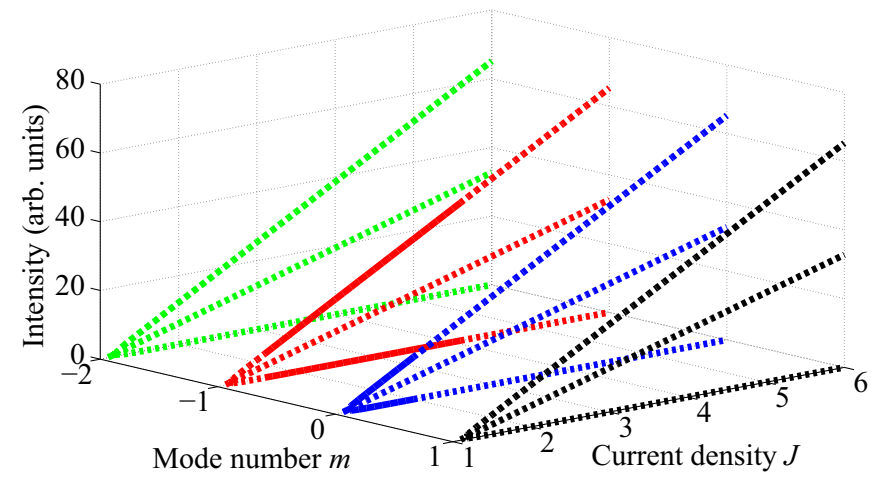

FIG. 7. (Color online) Bifurcation diagrams for four modes for a SRL with the parameters shown in Table I except $\mathcal{D}=2.32 \mathrm{~cm}^{2} \mathrm{~s}^{-1}$. There is a short region of longitudinal mode bistability between modes $m=0$ and $m=-1$; however, both modes become unstable at different currents and finally there is a multimode instability.

none of the modes is stable and the instability develops in good agreement with the numerical simulations.

Additional insight into this instability can be gained by examining the eigenvalue and eigenvector spectra for the different modes. Recall that the imaginary part of the eigenvalue gives the frequency of the perturbation eigenvector in the reference frame of the mode considered (see Appendix A for details). Also, the real part of the eigenvalue determines the stability of the perturbation: Eigenvalues with a positive real part correspond to unstable perturbation eigenvectors. Finally, the eigenvector determines whether the perturbation is copropagating or counterpropagating with the mode considered.

Figure 8 displays the dominant part of the eigenvalue spectra for monochromatic UNI states corresponding to modes $m=0, m=-1$, and $m=-2$ obtained at $J=5$. It can be seen [Fig. 8(a)] that mode $m=0$ is unstable with respect to a counterpropagating solution at frequency $\operatorname{Im}\{\lambda\} \approx 2 \pi$, i.e., against perturbations on mode $m^{\prime}=-1$. Similarly, mode $m=-1$ [Fig. 8(b)] is unstable with respect to a counterpropagating solution at frequency $\operatorname{Im}\{\lambda\} \approx 2 \pi$, i.e., against perturbations on $m^{\prime}=-2$. On the other hand, the
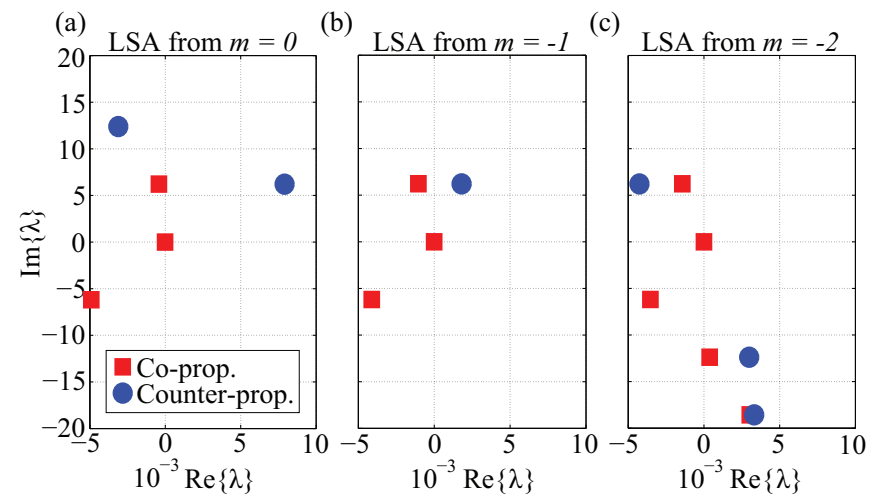

FIG. 8. (Color online) Eigenvalue spectra corresponding to performing numerically the LSA of Eqs. (1)-(4) for the monochromatic solutions for modes $m=0$ (a), $m=-1$ (b), and $m=-2$ (c) at $J=5$. We use the associated eigenvectors in order to find to what direction of emission the eigenvalues correspond.

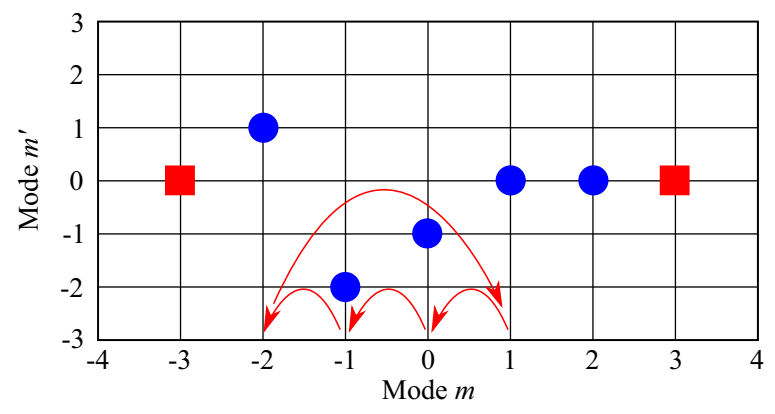

FIG. 9. (Color online) Most unstable perturbation mode $m^{\prime}$ for UNI solutions in mode $m$ at $J=5$. Blue circles denote counterpropagating modes and red squares indicate copropagating modes. The red arrows indicate the cycle sequence arising from the instability.

monochromatic solution for $m=-2$ [Fig. 8(c)] is unstable with respect to perturbations - both counterpropagating and copropagating - at frequencies $\operatorname{Im}\{\lambda\} \approx-4 \pi$ and $-6 \pi$, i.e., against perturbations at modes $m^{\prime}=0$ and $m^{\prime}=1$.

The cyclic switching character of the instability can be revealed by plotting for each mode $m$ the most unstable perturbation mode $m^{\prime}$, as shown in Fig. 9. It is seen that mode $m=1$ is most unstable against mode $m^{\prime}=0$; in turn, $m=0$ is most unstable against $m^{\prime}=-1$, mode $m=-1$ is most unstable against $m^{\prime}=-2$, and mode $m=-2$ is most unstable against $m^{\prime}=1$, and in all these cases the most unstable mode is counterpropagating with respect to $m$. Instead, modes with $|m| \geqslant 3$ are most unstable against copropagating perturbations at mode $m^{\prime}=0$, but the counterpropagating perturbations are also unstable. This particular structure explains the modal cycle from blue to red accompanied by a directional switching: if one randomly perturbs a UNI directional solution on mode $m=1$, the solution will initially approach mode $m=0$, then pass on to mode $m=-1$, further progress toward mode $m=-2$, and then jump back toward the original state; moreover, during this process, each modal jump is accompanied by a directional reversal. The same holds for UNI solutions on mode $m=0, m=-1$, or $m=-2$, and these characteristics explain qualitatively well the observed dynamics, schematically depicted with arrows in Fig. 9. However, it is worth remarking that mode $m=1$ contributes quite little to the observed dynamics. This can be explained by noting that mode $m=1$ is strongly unstable (its dominant eigenvalue has $\operatorname{Re}\{\lambda\} \approx 1.5 \times 10^{-2}$, which is twice that of the other modes in the cycle); as a consequence, the dynamics will be strongly pushed apart from $m=1$ toward $m=0$, and therefore it will have a short residence time, if any.

Yet, the prevalence of counterpropagating perturbations over copropagating disturbances may seem quite surprising, because counterpropagating waves generate a carrier grating that does not exist for copropagating waves; hence it may appear that diffusion should have a stronger impact on the former. The physical origin for this fact can be further elucidated by considering the ideal case of a pure SRL, i.e., when reflections are neglected, $r_{ \pm}=0$. Assuming unidirectional operation on a given mode $m=s$, the monochromatic solutioncharacterized by $E_{+}^{s} \neq 0, P_{+}^{s} \neq 0, N_{0}^{s} \neq 0$, and $E_{-}^{s}=P_{-}^{s}=$ $N_{ \pm 2}^{s}=0$ - can be determined as detailed in Appendix B. The LSA of the perturbations around such a solution decouples 
counterpropagating from copropagating perturbations (see Appendix B for the derivation). In the reference frame of the clockwise UNI lasing solution, counterpropagating perturbations are governed by Eqs. (B23)-(B25),

$$
\begin{gathered}
\partial_{t} e_{-}-\partial_{z} e_{-}=-\alpha_{i} e_{-}+i p_{-}, \\
\partial_{t} n_{-2}=-\left[R^{\prime}\left(N_{0}^{s}\right)+\eta\right] n_{-2}-i\left(p_{-} E_{+}^{s *}-e_{-} P_{+}^{s *}\right),
\end{gathered}
$$

and

$$
\begin{aligned}
p_{-}= & \int_{0}^{\infty} d t^{\prime} e^{i \omega_{s} t^{\prime}}\left[\chi\left(t^{\prime}, N_{0}^{s}\right) e_{-}\left(z, t-t^{\prime}\right)\right. \\
& \left.+\partial_{N} \chi\left(t^{\prime}, N_{0}^{s}\right) n_{-2}\left(z, t-t^{\prime}\right) E_{+}^{s}\right] .
\end{aligned}
$$

Copropagating perturbations, instead, are ruled by Eqs. (B38)(B40),

$$
\begin{gathered}
\partial_{t} e_{+}+\partial_{z} e_{+}=\left(i \omega_{s}-i k_{s}-\alpha_{i}\right) e_{+}+i p_{+}, \\
\partial_{t} n_{0}=-R^{\prime}\left(N_{0}^{s}\right) n_{0}-i\left(P_{+}^{s} e_{+}^{*}+p_{+} E_{+}^{s *}-\text { c.c. }\right),
\end{gathered}
$$

and

$$
\begin{aligned}
p_{+}= & \int_{0}^{\infty} d t^{\prime} e^{i \omega_{s} t^{\prime}}\left[\chi\left(t^{\prime}, N_{0}^{s}\right) e_{+}\left(z, t-t^{\prime}\right)\right. \\
& \left.+\partial_{N} \chi\left(t^{\prime}, N_{0}^{s}\right) n_{0}\left(z, t-t^{\prime}\right) E_{+}^{s}\right] .
\end{aligned}
$$

There are remarkable differences between the two sets of equations. For copropagating disturbances, the linearized equations describe the generalized relaxation oscillations in a multimode system and they involve slowly spatially evolving carrier pulsation at a spatial frequency equal to $2 \pi m$ with $m=0, \pm 1, \pm 2, \ldots$. As discussed in Appendix B, this requires that perturbations $\ell$ modes above $s$ are coupled to perturbations $\ell$ modes below $s$ by carrier-mediated FWM. The reason is that two optical modes, say, $s$ and $s-\ell$, create a modulation wave in the carrier density at frequency $\ell$ that is coupled back into the optical field through the active medium polarization in order to generate an additional side mode at frequency $s+\ell$.

Instead, the (linearized) evolution of counterpropagating disturbances does not depend on perturbations in the total carrier density $N_{0}$ but only on those for the amplitude of the carrier grating, $n_{2}$. The equation for the carrier grating amplitude has a source term such that two counterpropagating plane waves at different frequencies create a population grating that is going to slide along the cavity at a speed given by the frequency difference between the two modes [31,32]. This sliding grating does not generate new spatial or temporal frequencies for the field through its interaction with the active medium.

As a consequence, counterpropagating perturbations can grow more easily than copropagating perturbations because for the latter the interaction is mediated by a carrier density wave that imposes the requirement that the energy of the perturbation has to be shared between two different modes which in addition have to maintain a precise phase relation. In other words, creating a carrier grating that slides across the cavity at a given speed is energetically favorable as compared to generating an equivalent pulsation in the carrier density, at least for frequencies in the vicinity of the gain peak.
It is worth remarking that, even in the case of an ideal SRL, the eigenvalue equations that result for both copropagating and counterpropagating solutions are strongly nonlinear. However, simplified eigenvalue equations can be obtained when considering the UFL, i.e., $t_{ \pm}=1$. In this limit-where the field and the polarization are pure waves of constant amplitude, and the carrier density is constant along the cavity-it is possible to analytically perform the LSA of the monochromatic solutions as in [45] (see Appendix B), since the spatial dependence of the problem reduces to a pure phase factor $e^{i k_{s} z}$. Moreover, for a given mode $m=\ell$ (with respect to the UNI lasing solution), the eigenvalues must be close to the modal frequency.

Thus, in the UFL the eigenvalue equation for counterpropagating perturbations becomes a second-order polynomial for $\lambda^{\prime}=\lambda+2 i \pi \ell$

$\lambda^{\prime}=i\left(\tilde{\chi}_{\ell}-\tilde{\chi}_{s}\right)+\frac{\partial_{N} \tilde{\chi}_{\ell}\left(\tilde{\chi}_{\ell}-\tilde{\chi}_{s}^{*}\right)\left|E_{+}^{s}\right|^{2}}{R^{\prime}\left(N_{0}^{s}\right)+\eta+i \partial_{N} \tilde{\chi}_{\ell}\left|E_{+}^{s}\right|^{2}+\lambda^{\prime}-2 i \pi \ell}$,

where $\tilde{\chi}_{\ell}, \partial_{N} \tilde{\chi}_{\ell}$, and $\tilde{\chi}_{s}$ can be found in Appendix B. Most of the physics of this instability can be understood by inspecting Eq. (14). The first term on the right-hand side (RHS) corresponds to the gain difference between modes. Since one operates initially at the gain peak, this difference is negative, thereby ensuring stable operation with respect to side mode perturbations. The second term in the RHS is the one responsible for the asymmetric modal switching. The numerator contains the differential gain $\partial_{N} \tilde{\chi}_{n}$ which contains the so-called $\alpha$ factor, while the denominator also contains a complex response that consists of the balance between the modal separation $2 i \pi \ell$ and the variation of $\tilde{\chi}$ with the carrier density times the intensity of the field. Clearly, an instability can be promoted for $\ell<0$ and inhibited for $\ell>0$. Notice also in this complex denominator the presence of the half-wavelength diffusion factor $\eta$ which makes this "two-wave-mixing" term small.

For a copropagating perturbation, one has to take into account the perturbation in the mode of interest and the perturbation of the mode that it is created by FWM with the monochromatic state [45]. Finally, in the UFL a third-order polynomial for the eigenvalue $\lambda^{\prime}$ is obtained,

$$
\begin{aligned}
& {\left[\lambda^{\prime}-i\left(\tilde{\chi}_{-\ell}-\tilde{\chi}_{s}\right)-\frac{\partial_{N} \tilde{\chi}_{-\ell}\left(\tilde{\chi}_{-\ell}-\tilde{\chi}_{s}^{*}\right)\left|E_{+}^{s}\right|^{2}}{\lambda^{\prime}+Y_{\ell}}\right]} \\
& \times\left[\lambda^{\prime}+i\left(\tilde{\chi}_{\ell}^{*}-\tilde{\chi}_{s}^{*}\right)-\frac{\partial_{N} \tilde{\chi}_{\ell}^{*}\left(\tilde{\chi}_{\ell}^{*}-\tilde{\chi}_{s}\right)\left|E_{+}^{s}\right|^{2}}{\lambda^{\prime}+Y_{\ell}}\right] \\
& -\frac{\partial_{N} \tilde{\chi}_{-\ell} \partial_{N} \tilde{\chi}_{\ell}^{*}\left(\tilde{\chi}_{\ell}^{*}-\tilde{\chi}_{s}\right)\left(\tilde{\chi}_{-\ell}-\tilde{\chi}_{s}^{*}\right)\left|E_{+}^{s}\right|^{4}}{\left(\lambda^{\prime}+Y_{\ell}\right)^{2}}=0,
\end{aligned}
$$

where $Y_{\ell}=2 i \pi \ell+R^{\prime}\left(N_{0}^{s}\right)-i\left(\partial_{N} \tilde{\chi}_{\ell}^{*}-\partial_{N} \tilde{\chi}_{-\ell}\right)\left|E_{+}^{s}\right|^{2}$. Although Eq. (15) is more involved than Eq. (14), we notice that the first term is composed of the product of two terms similarly to Eq. (14). Figure 10 show the eigenvalues $\lambda$ obtained from Eqs. (14) and (15). Comparing Fig. 10 with Fig. 8, we can see that the unstable values are recovered; however, there are some differences that come from the assumptions used in the derivation of Eqs. (14) and (15). Still, the good qualitative agreement makes such approximate analytical expressions 


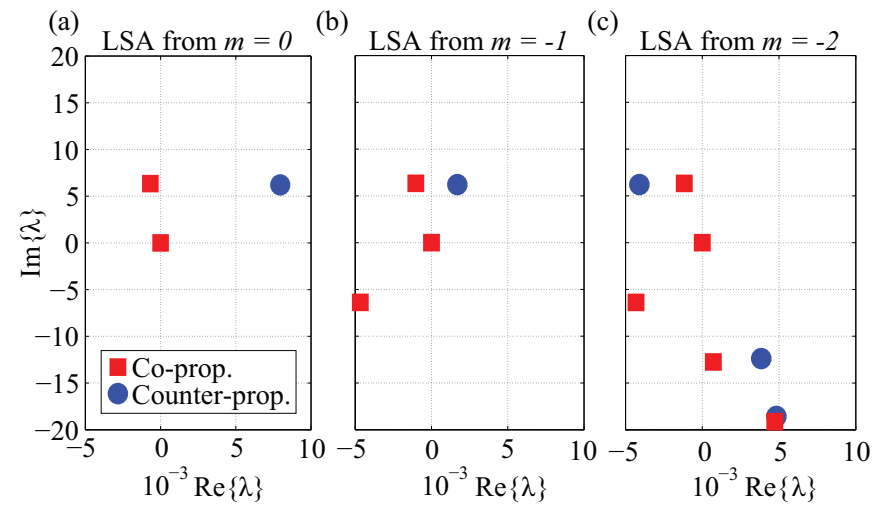

FIG. 10. (Color online) Eigenvalue spectra obtained from Eqs. (14) and (15) for the monochromatic solutions for modes $m=0$ (a), $m=-1$ (b), and $m=-2$ (c) at $J=5$.

useful to understand the underlying mechanism of the modal instability. From an extensive parameter study, we found that the instability with respect to copropagating solutions, like the one studied in [26], is always found for parameter values for which the system already became unstable with respect to directional reversals. This suggests that this modal instability mechanism should be the one dominantly observed experimentally.

Finally, we remark that the numerical results for the switching instability in Fig. 4 and the corresponding LSA (bifurcation diagrams and eigenvalue and eigenvector spectra) suggest the hypothesis of a heteroclinic orbit connecting the laser modes. The eigenvectors are pointing exactly in the direction of the red adjacent modes except for the last mode in the sequence, whose most unstable eigenvector points to the blue mode $m=1$, thereby enforcing the periodic behavior of the modal sequence. However, performing the continuation of such a heteroclinic connection with a fully spatially resolved TWM would represent a tremendous technical challenge.

Nevertheless, heteroclinic orbits are usually quite sensitive to noise; hence we have studied how the characteristics of this switching instability change with the level's spontaneousemission noise. On increasing the noise amplitude $\beta$, the behavior shown in Figs. 4 and 6 is conserved, but the duration of the switching and the period of the full oscillation fluctuate. In the absence of spontaneous emission noise $(\beta=0)$, the behavior changes considerably (see Fig. 11): The directional switching and the modal switching are not completed and there are more modes involved in the dynamics due to FWM. The results of Fig. 11 indicate that a small-amplitude limit cycle involving modal amplitude oscillation on the slow time scale of 50 ns coexists with the large-amplitude limit cycle that consists of multimode directional reversals. This hypothesis is supported by the fact that starting a simulation in the instability shown in Fig. 4 and removing the noise does not lead to the behavior shown in Fig. 11. The behavior shown in Fig. 11 is achieved only by starting from a noisy initial condition and performing the simulation with $\beta=0$. From the general point of view of nonlinear dynamics, one plausible scenario would be that a weakly attracting, small-amplitude limit cycle coexists with the large-amplitude heteroclinic orbit and they are separated by an unstable cycle that plays the role of the

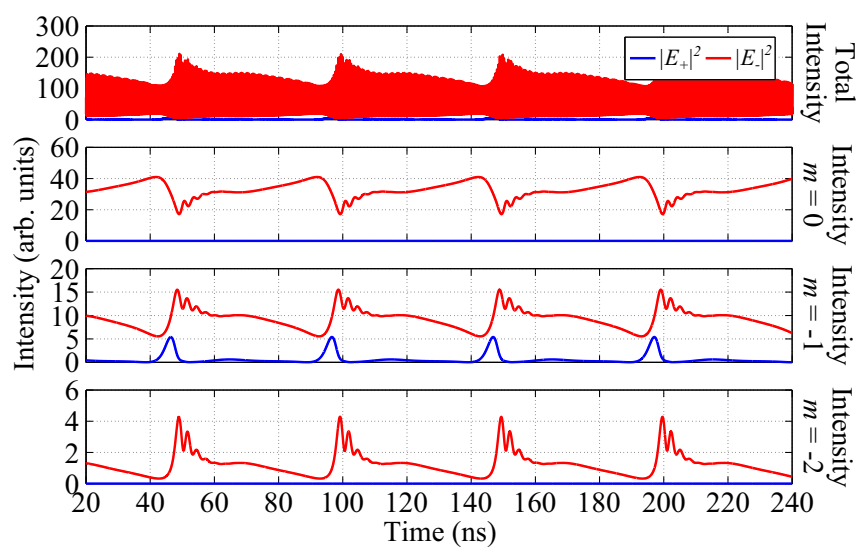

FIG. 11. (Color online) Effect of the absence of spontaneousemission noise in the longitudinal mode switching instability shown in Fig. 4. $J=5$. Parameters as in Table I except $\mathcal{D}=2.32 \mathrm{~cm}^{2} \mathrm{~s}^{-1}$ and $\beta=0$.

separatrix in phase space, but which is easily crossed in the presence of noise.

\section{CONCLUSIONS}

We have investigated theoretically and numerically the multimode dynamics in SRLs by performing a bifurcation analysis of a spatiotemporal TWM with the gain of a semiconductor QW [21]. Our bifurcation analysis extends and generalizes the results obtained in [28] for the case of a two-level atom active medium. Our investigation of the longitudinal mode multistability in SRLs has led us to find a multimode instability that leads to a dynamical regime where the lasing frequency jumps periodically from one mode to the next. The jumping sequence proceeds, at low frequency, from the bluest to the reddest part of the spectrum, and each modal jump is accompanied by a directional reversal. We have identified the modal instability mechanism to be of a similar nature to the one found in [26], which consists of the interplay between the carrier beatings, the asymmetry of the semiconductor gain curve and the $\alpha$ factor. However, this behavior is found here for much lower bias current due to the extra degree of freedom brought by the directional bistability of the SRLs. We have also found that the spontaneous emission noise plays a crucial role in the mechanism inducing the directional and modal switching. In the absence of spontaneous emission the switching is not achieved, and the system is capable only of showing multimode dynamics induced by FWM. This supports the idea of a low-amplitude limit cycle that coexists with a large-amplitude heteroclinic connection between the monomode solutions. This is not a noise-induced instability, which is against the concept of deterministic bifurcation and cannot be explained by our LSA. Although we were not able to perform a numerical continuation of such a heteroclinic connection, such a hypothesis was supported by the analysis of the most unstable eigenvectors around the monomode solutions. Finally, we gave quasiquantitatively correct approximations to the eigenvalues that define the boundary of the unstable regions which allowed us to contrast the instability thresholds for copropagating and contrapropagating perturbations. Since 
this directional instability seems to appear always before the copropagating one in ring lasers, we believe its observation to be possible, especially in long-wavelength SRLs. Finally, this analysis demonstrates that even the off modes that do not participate in the dynamics can strongly influence the LSA of the lasing modes. For instance a two-mode rate-equation model that considered a strong and a weak mode would be essentially incorrect, unless the two modes correspond to opposite lasing directions. In the case of a strongly multimode regime that consists of $N$ modes, a model that followed the multimode rate-equation approach would need at least $N / 2$ modes on each side of the spectrum in order to correctly assess the dynamics.

\section{ACKNOWLEDGMENTS}

J.J. acknowledges financial support from a Ramón y Cajal Fellowship. J.J. and S.B. acknowledge financial support from project RANGER (Grant No. TEC2012-38864-C03-01) and from the Direcció General de Recerca, Desenvolupament Tecnològic i Innovació de la Conselleria d'Innovació, Interior i Justícia del Govern de les Illes Balears cofunded by European Union FEDER funds.

\section{APPENDIX A: NUMERICAL BIFURCATION ANALYSIS}

\section{Monochromatic solutions}

In our past work [28] the simplicity of the two-level atom description allowed us to find the monochromatic solutions of the system by using a shooting method for the electric fields fulfilling the boundary conditions, while solving a linear system of equations for the material variables at each point. However, the monochromatic solutions for the TWM described by Eqs. (1)-(4) cannot be found in the same way, because the complexity of the system has increased due to the nonlinearity of the QW response, involving a convolution, and the use of DAEs; thus we are dealing with a highly multidimensional nonlinear problem that can be difficult to solve unless a good guess solution is provided. So, as described in Sec. II C we use the eigenvalues obtained from performing the LSA of the off solution as a guess for a Newton-Raphson solver obtaining the monochromatic solutions

Using this procedure allows us to construct bifurcation diagrams like the one shown in Fig. 12 for mode $m=0$ corresponding to parameter set shown in Table I. One can see that the complete L-I curve is recovered [2]. The SRL starts to

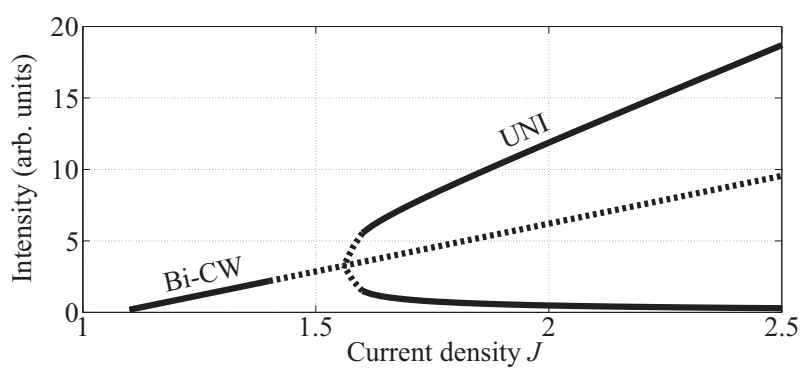

FIG. 12. Bifurcation diagram of the mode $m=0$ for the parameter set from Table I. The solid (dotted) black lines indicate stable (unstable) solutions for the branches Bi-CW and UNI. The unstable region around $J=1.5$ corresponds to the AO regime.
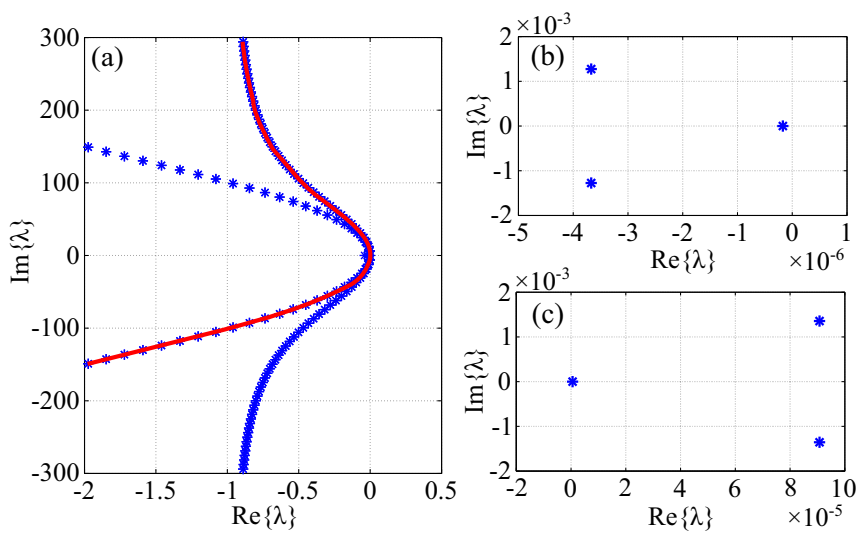

FIG. 13. (Color online) Eigenvalue spectra corresponding to Fig. 12 at $J=1.3$ (a),(b), and $J=1.4$ (c). The red line indicates the shape of the gain curve. (b),(c) Onset of the Hopf bifurcation by the crossing of the $\operatorname{Im}\{\lambda\}$ axis of two complex conjugate eigenvalues. The other eigenvalue shown corresponds to phase invariance.

lase bidirectionally (Bi-CW), then there is the onset of a Hopf bifurcation (see Fig. 13 for details) that leads to the AO regime, and finally the pitchfork bifurcation that takes place inside the AO regime changes its stability and leads to the UNI regime.

Figure 12 is completely equivalent to Fig. 2(c) in [46] which was obtained from the bifurcation analysis of a reduced twomode model for single-longitudinal-mode SRLs. In our case the linewidth enhancement factor $\alpha_{H}=1.16$ and we can think that $\phi_{k}$ comes mainly from the complex reflection coefficient $r_{ \pm}$; then $\phi_{k}=1.37 \mathrm{rad}$. Our analysis is in agreement with the one performed in [46] in the case of single-mode operation and in the UFL.

\section{Linear stability analysis}

The method used to perform the LSA of the system of Eqs. (1)-(4) is based in the fact that the TWM is written in the time domain. This allows us to use the temporal map $\vec{V}_{j+1}=$ $\vec{U}\left(h, \vec{V}_{j}\right)$ formed by the equations that are used to perform the numerical integration [36]. The temporal map advances the state vector $\vec{V}$ by a time step $h$ while verifying the CFL condition and canceling numerical dissipation [41].

Considering all possible perturbations of $\vec{V}$, one finds the matrix $\mathbf{M}=\partial \vec{U} / \partial \vec{V}$ representing the linear operator governing the time evolution for the perturbations around one given monochromatic solution: $E_{ \pm}^{s t}\left(z, \Delta t_{M}\right), P_{ \pm}^{s t}\left(z, \Delta t_{D}\right), N_{0}^{s t}(z)$, and $N_{ \pm 2}^{s t}(z)$, where $\Delta t_{M}$ and $\Delta t_{D}$ represent the time intervals associated with the convolution and the decimation factor, respectively. Numerically, we separate the problem into real and imaginary parts; then our system has a number of independent variables $\mathcal{N}=K(4 M+3)+4 D(K-2)+4(D+1)$ where $K=(N-1) / D+1$. In our case $\mathcal{N}=3423$. Here we have taken into account that the carrier density is almost constant in the time span of the convolution kernel calculation (over a few hundred femtoseconds) which allows us to perform the approximation $N_{0}(z, r)=N_{0}(z, t)$ and $N_{ \pm 2}(z, r)=N_{ \pm 2}(z, t)$ in Eq. (4). To obtain the evolution operator $\mathbf{M}$, an $\mathcal{N} \times \mathcal{N}$ matrix, one calculates each row by introducing a perturbation, i.e., one of the $\mathcal{N}$ variables is set to 1 whereas the others are zero. 
Then this state is evolved over one time step according to the numerical algorithm in [36] and taking into account the monochromatic solutions previously calculated. This process is repeated for all variables, obtaining M. One finally computes the $\mathcal{N}$ Floquet multipliers $z_{\mathcal{N}}$ of $\mathbf{M}$ via a $\mathrm{QR}$ decomposition method, which determines the eigenvalues as $\lambda_{\mathcal{N}}=h^{-1} \ln z_{\mathcal{N}}$. As usual if one of these computed eigenvalues has a positive real part, then one concludes that this monochromatic solution is unstable. If none of them has a positive real part, then the monochromatic solution is stable.

Due to the separation of the variables into real and imaginary parts, we obtain two eigenvalue spectra as shown in Fig. 13(a), one corresponding to the gain curve (indicated with a red line) and the other to the complex conjugates. In order to distinguish between the eigenvalues associated with the gain curve and their complex conjugates, we can think that in complex form a solution is formed by a steady state $\vec{E}^{s t}$ and an eigenperturbation $\vec{E}^{p}$ as

$$
\vec{E}=\vec{E}^{s t}+\epsilon \vec{E}^{p} e^{r t-i \omega t}
$$

where $\epsilon$ is a small complex amplitude, $r$ gives the grow or decay of the perturbation, and $\omega$ is the frequency of the perturbation. In our case we have separated the variables into real and imaginary parts for numerical purposes. Therefore, an equivalent expression to Eq. (A1) is

$$
\vec{V}=\vec{V}^{s t}+\left(\mu \vec{V}^{p} e^{\lambda t}+\text { c.c. }\right),
$$

where $\mu$ is a small complex amplitude and $\lambda=\lambda_{r}+i \lambda_{i}$ is one of the eigenvalues obtained numerically by the QR decomposition method. At each spatial point, we can think that a complex component $V_{1}$ is associated with the real part of the electric field and a component $V_{2}$ is associated with the imaginary part of the electric field (depending on the direction of emission of the eigenvector associated with the eigenvalue investigated, we will take into account the clockwise or the counterclockwise electric field). Thus we can write their corresponding eigenvector components as

$$
\begin{aligned}
& V_{1}^{p}=\left|V_{1}^{p}\right| e^{i \phi_{1}}, \\
& V_{2}^{p}=\left|V_{2}^{p}\right| e^{i \phi_{2}} .
\end{aligned}
$$

We define $\mu=e^{-i \phi_{1}}$ and we use Eq. (A2) in each component of our solution, obtaining

$$
\begin{gathered}
V_{1}=V_{1}^{s t}+2\left|V_{1}^{p}\right| e^{\lambda_{r} t} \cos \left(\lambda_{i} t\right) \\
V_{2}=V_{2}^{s t}+2\left|V_{2}^{p}\right| e^{\lambda_{r} t} \cos \left(\lambda_{i} t+\phi_{2}-\phi_{1}\right) .
\end{gathered}
$$

As we are interested in knowing the orientation of the rotation, we take the derivatives of $V_{1}$ and $V_{2}$ at $t=0$, neglecting their real parts which are not associated with the rotation; hence

$$
\begin{gathered}
\left.\partial_{t} V_{1}\right|_{t=0}=0, \\
\left.\partial_{t} V_{2}\right|_{t=0} \sim \sin \left(\phi_{1}-\phi_{2}\right),
\end{gathered}
$$

and the sign of $\sin \left(\phi_{1}-\phi_{2}\right)$ give us the sense of the rotation. Therefore, we compute the resulting eigenvalue as $\lambda=$ $\operatorname{Re}\{\lambda\}+i \operatorname{Im}\{\lambda\} \operatorname{sgn}(x)$, where $x=\sin \left(\phi_{1}-\phi_{2}\right)$. This process has been used to obtain Fig. 8 and the red line in Fig. 13(a), which shows the typical gain asymmetry characteristic of the QW material. We notice that in order to be as accurate as possible in the calculation of the eigenvalues, we perform a change of the frequency reference frame in the lasing solutions, which explains why the maximum of the gain curve is at $\operatorname{Im}\{\lambda\}=0$. It has also to be noticed that, in using this approach for computing the stability of a monochromatic solution different from the off solution, we obtain a zero eigenvalue corresponding to the phase invariance of the system.

Figures 13(b) and 13(c) show the eigenvalues closest to $\operatorname{Im}\{\lambda\}=0$ for two different values of $J, J=1.3$ and $J=1.4$. In both cases, a zero eigenvalue is obtained, which reflects phase invariance. Next to it, a pair of complex conjugate eigenvalues is seen in each panel. If we could continuously follow this pair of eigenvalues as the current is increased, we would see that they cross the imaginary axis at $J \simeq 1.33$, indicating the onset of a Hopf bifurcation that consists in the excitation of AOs by an undamping of the relaxation oscillations around the $\mathrm{Bi}-\mathrm{CW}$ solution. Following this procedure, we can construct the bifurcation diagrams for the dominant modes of a SRL with the parameters in Table I shown in Fig. 3.

\section{APPENDIX B: ANALYTICAL LSA FOR AN IDEAL SRL}

An ideal SRL is characterized by having no reflection at the output coupler, which in addition provides a lossless cavity. In this system, the boundary conditions (in the absence of injected fields) simply read

$$
E_{ \pm}(0, t)=E_{ \pm}(1, t)
$$

where the optical carrier frequency has been taken as that of the mode closest to the gain peak. In these case, the UFL applies and it is possible to perform the LSA analytically.

\section{Monochromatic solutions}

In order to perform the LSA analytically, the first step is to find the monochromatic solutions of Eqs. (1)-(4). These have the forms

$$
\begin{gathered}
E_{ \pm}(z, t)=E_{ \pm}^{s} e^{ \pm i k_{s} z-i \omega_{s} t} \\
P_{ \pm}(z, t)=P_{ \pm}^{s} e^{ \pm i k_{s} z-i \omega_{s} t} \\
N_{0}(z, t)=N_{0}^{s} \\
N_{ \pm 2}(z, t)=N_{ \pm 2}^{s} e^{ \pm 2 i k_{s} z}
\end{gathered}
$$

where $k_{s}=2 \pi s$ is the wave vector of the monochromatic solution (or mode) $m=s . E_{ \pm}^{s}, P_{ \pm}^{s}, N_{0}^{s}$, and $N_{ \pm 2}^{s}$ define a monochromatic solution that oscillates at angular frequency $\omega_{s}$, and they are given by

$$
\begin{gathered}
\left(i k_{s}-i \omega_{s}\right) E_{ \pm}^{s}=i P_{ \pm}^{s}-\alpha_{i} E_{ \pm}^{s}, \\
P_{ \pm}^{s}=\tilde{\chi}_{s} E_{ \pm}^{s}+\partial_{N} \tilde{\chi}_{s} E_{\mp}^{s} N_{ \pm 2}^{s}, \\
R\left(N_{0}^{s}\right)-J=-i\left(E_{+}^{s *} P_{+}^{s}+E_{-}^{s *} P_{-}^{s}-\text { c.c. }\right), \\
N_{ \pm 2}^{s}=-i \frac{P_{ \pm}^{s} E_{\mp}^{s *}-E_{ \pm}^{s} P_{\mp}^{s *}}{R^{\prime}\left(N_{0}^{s}\right)+\eta},
\end{gathered}
$$


where

$$
\begin{gathered}
\tilde{\chi}_{s}=\int_{0}^{\infty} \chi\left(t^{\prime}, N_{0}^{s}\right) e^{i \omega_{s} t^{\prime}} d t^{\prime}, \\
\partial_{N} \tilde{\chi}_{s}=\int_{0}^{\infty} \chi_{N}\left(t^{\prime}, N_{0}^{s}\right) e^{i \omega_{s} t^{\prime}} d t^{\prime} .
\end{gathered}
$$

Besides the trivial solution $E_{ \pm}^{s}=0=P_{ \pm}^{s}=N_{ \pm 2}^{2}$ and $J=$ $R\left(N_{0}^{s}\right)$, the system admits both bidirectional and unidirectional solutions. The former are difficult to determine analytically, and in addition it has been proven that they are unconditionally unstable [47,48]. Unidirectional solutions in the clockwise direction are of the form $E_{-}^{s}=P_{-}^{s}=N_{ \pm 2}^{s}=0$, with $E_{+}^{s} \neq 0$, $P_{+}^{s} \neq 0, N_{0}^{s} \neq 0$, and $\omega_{s}$ given by

$$
i k_{s}-i \omega_{s}=i \tilde{\chi}\left(\omega_{s}, N_{0}^{s}\right)-\alpha_{i}
$$

and

$$
\left|E_{+}^{s}\right|^{2}=\frac{J_{t h}-J}{2 \operatorname{Im}\left\{\tilde{\chi}_{s}\right\}}, \quad P_{+}^{s}=\tilde{\chi}_{s} E_{+}^{s}, \quad J_{t h}=R\left(N_{0}^{s}\right) .
$$

Obviously, an equivalent unidirectional solution exists that propagates in the opposite direction, which is simply obtained by exchanging the signs in the solution labels.

\section{Perturbation analysis of UNI solutions}

We consider the monochromatic unidirectional solution corresponding to mode $m=s$, which propagates in the clockwise direction, i.e., $E_{-}^{s}=P_{-}^{s}=N_{ \pm 2}^{s}=0, E_{+}^{s} \neq 0, P_{+}^{s} \neq$ $0, N_{0}^{s} \neq 0$, and $\omega_{s}$ determined by Eqs. (B12) and (B13). We disturb this solution with space- and time-dependent perturbations $e_{ \pm}(z, t), p_{ \pm}(z, t), n_{0}(z, t)$, and $n_{ \pm 2}(z, t)$, with $n_{-2}=n_{+2}^{*}$ and $n_{0}$ real. We introduce

$$
\begin{gathered}
E_{ \pm}(z, t)=E_{ \pm}^{s} e^{ \pm i k_{s} z-i \omega_{s} t}+e_{ \pm}(z, t), \\
P_{ \pm}(z, t)=P_{ \pm}^{s} e^{ \pm i k_{s} z-i \omega_{s} t}+p_{ \pm}(z, t), \\
N_{0}(z, t)=N_{0}^{s}+n_{0}(z, t), \\
N_{ \pm 2}(z, t)=N_{ \pm 2}^{s} e^{ \pm 2 i k_{s} z}+n_{ \pm 2}(z, t)
\end{gathered}
$$

in Eqs. (1)-(4), and linearizing around the UNI solution leads to

$$
\begin{gathered}
\partial_{t} e_{ \pm} \pm \partial_{z} e_{ \pm}=i p_{ \pm}-\alpha_{i} e_{ \pm} \\
\partial_{t} n_{0}=-R^{\prime}\left(N_{0}^{s}\right) n_{0}-i\left(P_{+}^{s} e^{i \Phi_{s}(z, t)} e_{+}^{*}\right. \\
\left.+p_{+} E_{+}^{s *} e^{-i \Phi_{s}(z, t)}-\text { c.c. }\right), \\
\partial_{t} n_{-2}=-\left[R^{\prime}\left(N_{0}^{s}\right)+\eta\right] n_{-2} \\
-i\left(p_{-} E_{+}^{s *}-e_{-} P_{+}^{s *}\right) e^{-i \Phi_{s}(z, t)}, \\
p_{+}(z, t)=\int_{0}^{\infty}\left[\chi\left(t^{\prime}, N_{0}^{s}\right) e_{+}(z, r)\right. \\
\left.+\chi_{N}\left(t^{\prime}, N_{0}^{s}\right) E_{+}^{s} n_{0}(z, r) e^{i \Phi_{s}(z, r)}\right]_{r=t-t^{\prime}} d t^{\prime},
\end{gathered}
$$

$$
\begin{aligned}
p_{-}(z, t)= & \int_{0}^{\infty}\left[\chi\left(t^{\prime}, N_{0}^{s}\right) e_{-}(z, r)\right. \\
& \left.+\chi_{N}\left(t^{\prime}, N_{0}^{s}\right) E_{+}^{s} n_{-2}(z, r) e^{i \Phi_{s}(z, r)}\right]_{r=t-t^{\prime}} d t^{\prime}
\end{aligned}
$$

where $\Phi_{s}(z, t)=k_{s} z-\omega_{s} t$. Perturbations associated with opposite propagation directions decouple from each other, which allows for a separate analysis.

\section{a. Counterpropagating perturbation}

Propagations that propagate in the counterclockwise direction evolve (in the linearized regime) according to

$$
\partial_{t} e_{-}-\partial_{z} e_{-}=i p_{-}-\alpha_{i} e_{-}
$$

$$
\begin{aligned}
\partial_{t} n_{-2}= & -\left[R^{\prime}\left(N_{0}^{s}\right)+\eta\right] n_{-2} \\
& -i\left(p_{-} E_{+}^{s *}-e_{-} P_{+}^{s *}\right) e^{-i \Phi_{s}(z, t)},
\end{aligned}
$$

$$
\begin{aligned}
p_{-}(z, t)= & \int_{0}^{\infty}\left[\chi\left(t^{\prime}, N_{0}^{s}\right) e_{-}(z, r)\right. \\
& \left.+\chi_{N}\left(t^{\prime}, N_{0}^{s}\right) E_{+}^{s} n_{-2}(z, r) e^{i \Phi_{s}(z, r)}\right]_{r=t-t^{\prime}} d t^{\prime}
\end{aligned}
$$

which do not depend on perturbations to the carrier density, but only on the amplitude of the carrier grating.

It is convenient to make the changes $e_{-}(z, t)=$ $a_{-}(z, t) e^{-i k_{s} z-i \omega_{s} t}, \quad p_{-}(z, t)=b_{-}(z, t) e^{-i k_{s} z-i \omega_{s} t}, \quad$ and $n_{-2}(z, t)=c_{-2}(z, t) e^{-2 i k_{s} z}$, which lead to

$$
\begin{gathered}
\partial_{t} a_{-}-\partial_{z} a_{-}=-i \tilde{\chi}_{s} a_{-}+i b_{-}, \\
\partial_{t} c_{-2}=-\left[R^{\prime}\left(N_{0}^{s}\right)+\eta\right] c_{-2}-i\left(b_{-} E_{+}^{s *}-a_{-} P_{+}^{s *}\right), \\
b_{-}(z, t)=\int_{0}^{\infty}\left[\chi\left(t^{\prime}, N_{0}^{s}\right) a_{-}\left(z, t-t^{\prime}\right)\right. \\
\left.+\chi_{N}\left(t^{\prime}, N_{0}^{s}\right) E_{+}^{s} c_{-2}\left(z, t-t^{\prime}\right)\right] d t^{\prime}
\end{gathered}
$$

where we have used Eq. (B12). Assuming a perturbation that corresponds to a cavity mode (i.e., whose spatial dependence is of the form $e^{i k_{\ell} z}$ with $k_{\ell}=2 \pi \ell$ ), the boundary conditions are automatically satisfied. Introducing the eigenvalue $\lambda$ for the temporal evolution, i.e.,

$$
\begin{aligned}
& a_{-}=a_{\ell}^{-} e^{\lambda t} e^{-i k_{\ell} z}, \\
& b_{-}=b_{\ell}^{-} e^{\lambda t} e^{-i k_{\ell} z}, \\
& n_{-2}=\gamma_{\ell} e^{\lambda t} e^{-i k_{\ell} z},
\end{aligned}
$$

one arrives at the equation for the eigenvalues $\lambda$,

$$
\lambda+i k_{\ell}=i\left(\tilde{\chi}_{\lambda}-\tilde{\chi}_{s}\right)+\frac{\partial_{N} \tilde{\chi}_{\lambda}\left(\tilde{\chi}_{\lambda}-\tilde{\chi}_{s}^{*}\right)\left|E_{+}^{s}\right|^{2}}{R^{\prime}\left(N_{0}^{s}\right)+\eta+i \partial_{N} \tilde{\chi}_{\lambda}\left|E_{+}^{s}\right|^{2}+\lambda},
$$

where

$$
\tilde{\chi}_{\lambda}=\int_{0}^{\infty} \chi\left(t^{\prime}, N_{0}^{s}\right) e^{i\left(\omega_{s}+i \lambda\right) t^{\prime}} d t^{\prime}
$$




$$
\partial_{N} \tilde{\chi}_{\lambda}=\int_{0}^{\infty} \partial_{N} \chi\left(t^{\prime}, N_{0}^{s}\right) e^{i\left(\omega_{s}+i \lambda\right) t^{\prime}} d t^{\prime}
$$

The eigenvalue equation for the counterpropagating perturbations cannot be analytically solved due to the complicated dependence on $\lambda$ of $\tilde{\chi}_{\lambda}$ and $\partial_{N} \tilde{\chi}_{\lambda}$, which describe the spectral dependence of the optical response of the QW material and its variation with carrier density, respectively. These magnitudes, however, vary in wavelength intervals typically much larger than the characteristic mode spacing; hence one can expect the eigenvalue $\lambda$ to be close to the modal frequency for mode $\ell$. Hence, defining $\lambda=\lambda^{\prime}-2 i \pi \ell-$ where $\lambda^{\prime}$ is smalland neglecting the effect of $\lambda^{\prime}$ in (B33) and (B34) allows Eq. (B32) to be rewritten as

$$
\lambda^{\prime}=i\left(\tilde{\chi}_{\ell}-\tilde{\chi}_{s}\right)+\frac{\partial_{N} \tilde{\chi}_{\ell}\left(\tilde{\chi}_{\ell}-\tilde{\chi}_{s}^{*}\right)\left|E_{+}^{s}\right|^{2}}{R^{\prime}\left(N_{0}^{s}\right)+\eta+i \partial_{N} \tilde{\chi}_{\ell}\left|E_{+}^{s}\right|^{2}+\lambda^{\prime}-2 i \pi \ell},
$$

which is a second-order polynomial in $\lambda^{\prime}$ quoted in the main text as Eq. (14) and where we have defined

$$
\begin{gathered}
\tilde{\chi}_{\ell}=\int_{0}^{\infty} \chi\left(t^{\prime}, N_{0}^{s}\right) e^{i\left(\omega_{s}+2 \pi \ell\right) t^{\prime}} d t^{\prime}, \\
\partial_{N} \tilde{\chi}_{\ell}=\int_{0}^{\infty} \partial_{N} \chi\left(t^{\prime}, N_{0}^{s}\right) e^{i\left(\omega_{s}+2 \pi \ell\right) t^{\prime}} d t^{\prime} .
\end{gathered}
$$

\section{b. Copropagating perturbations}

As discussed before, copropagating perturbations evolve according to

$$
\begin{gathered}
\partial_{t} e_{+}+\partial_{z} e_{+}=i p_{+}-\alpha_{i} e_{+} \\
\partial_{t} n_{0}=-R^{\prime}\left(N_{0}^{s}\right) n_{0}-i\left(P_{+}^{s} e^{i \Phi_{s}(z, t)} e_{+}^{*}\right. \\
\left.+p_{+} E_{+}^{s *} e^{-i \Phi_{s}(z, t)}-\text { c.c. }\right), \\
p_{+}(z, t)=\int_{0}^{\infty}\left[\chi\left(t^{\prime}, N_{0}^{s}\right) e_{+}(z, r)\right. \\
\left.+\chi_{N}\left(t^{\prime}, N_{0}^{s}\right) E_{+}^{s} n_{0}(z, r) e^{i \Phi_{s}(z, r)}\right]_{r=t-t^{\prime}} d t^{\prime}
\end{gathered}
$$

In this case, coupling of the field perturbations to the material occurs through the perturbations in the local carrier density, and the amplitude of the carrier grating does not play any role. This simply reflects that copropagating waves do not generate a carrier grating, but simply create a modulation of the carrier density on long spatial distances as compared to the wavelength due to their beating. Please note that the above equations generalize the so-called relaxation oscillations to a spatially extended system.

It is convenient to pass to the reference frame of the UNI solution by setting $e_{+}(z, t)=a_{+}(z, t) e^{i \Phi_{s}(z, t)}, p_{+}(z, t)=$ $b_{+}(z, t) e^{i \Phi_{s}(z, t)}$, which leads to

$$
\begin{gathered}
\partial_{t} a_{+}+\partial_{z} a_{+}=\left(i \omega_{s}-i k_{s}-\alpha_{i}\right) a_{+}+i b_{+}, \\
\partial_{t} n_{0}=-R^{\prime}\left(N_{0}^{s}\right) n_{0}-i\left(P_{+}^{s} a_{+}^{*}+b_{+} E_{+}^{s *}-\text { c.c. }\right),
\end{gathered}
$$

$$
\begin{aligned}
b_{+}= & \chi_{0} \int_{0}^{\infty} e^{i \omega_{s} t^{\prime}}\left[\chi\left(t^{\prime}, N_{0}^{s}\right) a_{+}(z, r)\right. \\
& \left.+\chi_{N}\left(t^{\prime}, N_{0}^{s}\right) n_{0}(z, r) E_{+}^{s}\right] d t^{\prime},
\end{aligned}
$$

together with the corresponding equations for $a_{+}^{*}$ and $b_{+}^{*}$.

It is worth remarking that in these equations, perturbations cannot be on a single mode only: Disturbances $\ell$ modes above the monochromatic solution are tied to perturbations $\ell$ modes below via FWM mediated by the carrier density perturbation [45]. Therefore both have to be taken into account. For the sake of simplicity in the notation we suppose $s=0$; hence we take

$$
\begin{aligned}
& a_{+}=a_{-\ell}(t) e^{-i k_{\ell} z}+a_{\ell}(t) e^{i k_{\ell} z}, \\
& b_{+}=b_{-\ell}(t) e^{-i k_{\ell} z}+b_{\ell}(t) e^{i k_{\ell} z}, \\
& n_{0}=c_{-\ell}(t) e^{-2 i k_{\ell} z}+c_{\ell}(t) e^{2 i k_{\ell} z},
\end{aligned}
$$

which yields

$$
\begin{aligned}
\partial_{t} a_{-\ell}= & \left(2 i \pi \ell+i \omega_{s}-i k_{s}-\alpha_{i}\right) a_{-\ell}+i b_{-\ell}, \\
\partial_{t} a_{\ell}= & \left(-2 i \pi \ell+i \omega_{s}-i k_{s}-\alpha_{i}\right) a_{\ell}+i b_{\ell}, \\
\partial_{t} c_{-\ell}= & -R^{\prime}\left(N_{0}^{s}\right) c_{-\ell}-i\left(P_{+}^{s} a_{\ell}^{*}\right. \\
& \left.+b_{-\ell} E_{+}^{s *}-P_{+}^{s *} a_{-\ell}-b_{\ell}^{*} E_{+}^{s}\right), \\
\partial_{t} c_{\ell}= & -R^{\prime}\left(N_{0}^{s}\right) c_{\ell}-i\left(P_{+}^{s} a_{-\ell}^{*}\right. \\
& \left.+b_{\ell} E_{+}^{s *}-P_{+}^{s *} a_{m}-b_{-\ell}^{*} E_{+}^{s}\right)
\end{aligned}
$$

and

$$
\begin{aligned}
b_{-\ell}= & \int_{0}^{\infty} e^{i \omega_{s} t^{\prime}}\left[\chi\left(t^{\prime}, N_{0}^{s}\right) a_{-\ell}\left(t-t^{\prime}\right)\right. \\
& \left.+\chi_{N}\left(t^{\prime}, N_{0}^{s}\right) c_{-\ell}\left(t-t^{\prime}\right) E_{+}^{s}\right] d t^{\prime}, \\
b_{\ell}= & \int_{0}^{\infty} e^{i \omega_{s} t^{\prime}}\left[\chi\left(t^{\prime}, N_{0}^{s}\right) a_{\ell}\left(t-t^{\prime}\right)\right. \\
& \left.+\chi_{N}\left(t^{\prime}, N_{0}^{s}\right) c_{\ell}\left(t-t^{\prime}\right) E_{+}^{s}\right] d t^{\prime} .
\end{aligned}
$$

Clearly, $c_{-\ell}=c_{\ell}^{*}$, as expected since $n_{0}$ is real. Introducing the eigenvalue $\lambda$ as in the previous case and solving for the resulting system leads to

$$
\begin{aligned}
& {\left[\lambda-2 i \pi \ell-i\left(\tilde{\chi}_{\lambda}-\tilde{\chi}_{s}\right)-\frac{\partial_{N} \tilde{\chi}_{\lambda}\left(\tilde{\chi}_{\lambda}-\tilde{\chi}_{s}^{*}\right)\left|E_{+}^{s}\right|^{2}}{\lambda+Y_{\lambda}}\right]} \\
& \times\left[\lambda-2 i \pi \ell+i\left(\widehat{\chi}_{\lambda}-\tilde{\chi}_{s}^{*}\right)-\frac{\partial_{N} \widehat{\chi}_{\lambda}\left(\widehat{\chi}_{\lambda}-\tilde{\chi}_{s}\right)\left|E_{+}^{s}\right|^{2}}{\lambda+Y_{\lambda}}\right] \\
& -\frac{\partial_{N} \tilde{\chi}_{\lambda} \partial_{N} \widehat{\chi}_{\lambda}\left(\widehat{\chi}_{\lambda}-\tilde{\chi}_{s}\right)\left(\tilde{\chi}_{\lambda}-\tilde{\chi}_{s}^{*}\right)\left|E_{+}^{s}\right|^{4}}{\left(\lambda+Y_{\lambda}\right)^{2}}=0,
\end{aligned}
$$


where $Y_{\lambda}=R^{\prime}\left(N_{0}^{s}\right)-i\left(\partial_{N} \widehat{\chi_{\lambda}}-\partial_{N} \tilde{\chi}_{\lambda}\right)\left|A_{s}\right|^{2}$,

$$
\begin{gathered}
\tilde{\chi}_{\lambda}=\chi_{0} \int_{0}^{\infty} \chi\left(t^{\prime}, N_{0}^{s}\right) e^{i\left(\omega_{s}+i \lambda\right) t^{\prime}} d t^{\prime}, \\
\partial_{N} \tilde{\chi}_{\lambda}=\chi_{0} \int_{0}^{\infty} \partial_{N} \chi\left(t^{\prime}, N_{0}^{s}\right) e^{i\left(\omega_{s}+i \lambda\right) t^{\prime}} d t^{\prime}, \\
\widehat{\chi}_{\lambda}=\chi_{0} \int_{0}^{\infty} \chi^{*}\left(t^{\prime}, N_{0}^{s}\right) e^{-i\left(\omega_{s}-i \lambda\right) t^{\prime}} d t^{\prime}, \\
\partial_{N} \widehat{\chi}_{\lambda}=\chi_{0} \int_{0}^{\infty} \partial_{N} \chi^{*}\left(t^{\prime}, N_{0}^{s}\right) e^{-i\left(\omega_{s}-i \lambda\right) t^{\prime}} d t^{\prime},
\end{gathered}
$$

and we have used Eq. (B12) and that $P_{+}^{s}=\tilde{\chi}_{s} E_{+}^{s}$.

As in the previous section, the eigenvalue must be close to the modal frequency, so we define $\lambda=\lambda^{\prime}-2 i \pi \ell$ and we approximate

$$
\tilde{\chi}_{\lambda} \simeq \chi_{0} \int_{0}^{\infty} \chi\left(t^{\prime}, N_{0}^{s}\right) e^{i\left(\omega_{s}-2 \pi \ell\right)} d t^{\prime} \equiv \tilde{\chi}_{-\ell}
$$

$$
\widehat{\chi}_{\lambda} \simeq \chi_{0} \int_{0}^{\infty} \chi^{*}\left(t^{\prime}, N_{0}^{s}\right) e^{-i\left(\omega_{s}+2 \pi \ell\right)} d t^{\prime} \equiv \tilde{\chi}_{\ell}^{*},
$$

and accordingly for their derivatives with respect to carrier density. Hence we finally obtain the approximate eigenvalue equation

$$
\begin{gathered}
{\left[\lambda^{\prime}-i\left(\tilde{\chi}_{-\ell}-\tilde{\chi}_{s}\right)-\frac{\partial_{N} \tilde{\chi}_{-\ell}\left(\tilde{\chi}_{-\ell}-\tilde{\chi}_{s}^{*}\right)\left|E_{+}^{s}\right|^{2}}{\lambda^{\prime}+Y_{\ell}}\right]} \\
\times\left[\lambda^{\prime}+i\left(\tilde{\chi}_{\ell}^{*}-\tilde{\chi}_{s}^{*}\right)-\frac{\partial_{N} \tilde{\chi}_{\ell}^{*}\left(\tilde{\chi}_{\ell}^{*}-\tilde{\chi}_{s}\right)\left|E_{+}^{s}\right|^{2}}{\lambda^{\prime}+Y_{\ell}}\right] \\
-\frac{\partial_{N} \tilde{\chi}_{-\ell} \partial_{N} \tilde{\chi}_{\ell}^{*}\left(\tilde{\chi}_{\ell}^{*}-\tilde{\chi}_{s}\right)\left(\tilde{\chi}_{-\ell}-\tilde{\chi}_{s}^{*}\right)\left|E_{+}^{s}\right|^{4}}{\left(\lambda^{\prime}+Y_{\ell}\right)^{2}}=0,
\end{gathered}
$$

where $Y_{\ell}=2 i \pi \ell+R^{\prime}\left(N_{0}^{s}\right)-i\left(\partial_{N} \tilde{\chi}_{\ell}^{*}-\partial_{N} \tilde{\chi}_{-\ell}\right)\left|E_{+}^{s}\right|^{2}$. In this case Eq. (B60) is a third-order polynomial in $\lambda^{\prime}$ and it is given in the main text as Eq. (15).
[1] S. Yu and B. Li, in Proceedings of the Conference on Lasers and Electro-Optics Europe (CLEO EUROPE/EQEC) and 12th European Quantum Electronics Conference (IEEE Conference Publications, Munich, 2011).

[2] M. Sorel, G. Giuliani, A. Scirè, R. Miglierina, S. Donati, and P. J. R. Laybourn, IEEE J. Quantum Electron. 39, 1187 (2003).

[3] M. Sorel, P. J. R. Laybourn, A. Scirè, S. Balle, G. Giuliani, R. Miglierina, and S. Donati, Opt. Lett. 27, 1992 (2002).

[4] M. Sorel, P. J. R. Laybourn, G. Giuliani, and S. Donati, Appl. Phys. Lett. 80, 3051 (2002).

[5] L. Gelens, S. Beri, G. Van der Sande, G. Mezosi, M. Sorel, J. Danckaert, and G. Verschaffelt, Phys. Rev. Lett. 102, 193904 (2009).

[6] S. Fürst and M. Sorel, IEEE Photon. Technol. Lett. 20, 366 (2008).

[7] J. Javaloyes, A. Trita, G. Mezosi, F. Bragheri, I. Cristiani, G. Giuliani, M. Sorel, A. Scirè, and S. Balle, in Proceedings of the Conference on Lasers and Electro-Optics Europe (CLEO EUROPE/EQEC) and 11th European Quantum Electronics Conference (IEEE Conference Publications, Munich, 2009).

[8] Z. Wang, G. Yuan, X. Cai, G. Verschaffelt, J. Danckaert, Y. Liu, and S. Yu, IEEE Photon. Technol. Lett. 22, 1805 (2010).

[9] J. Sakaguchi, T. Katayama, and H. Kawaguchi, IEEE J. Quantum Electron. 46, 1526 (2010).

[10] M. T. Hill, H. J. S. Dorren, T. de Vries, X. J. M. Leijtens, J. H. den Besten, B. Smalbrugge, Y. S. Oei, H. Binsma, G. D. Khoe, and M. K. Smit, Nature (London) 432, 206 (2004).

[11] B. Li, M. Memon, G. Mezosi, Z. Wang, M. Sorel, and S. Yu, J. Opt. Commun. 30, 190 (2009).

[12] A. Trita, G. Mezosi, M. Latorre-Vidal, M. Zanola, M. Strain, F. Bragheri, M. Sorel, and G. Giuliani, IEEE J. Quantum Electron. 49, 877 (2013).

[13] S. Sunada, T. Harayama, K. Arai, K. Yoshimura, K. Tsuzuki, A. Uchida, and P. Davis, Opt. Express 19, 7439 (2011).

[14] R. M. Nguimdo, G. Verschaffelt, J. Danckaert, X. Leijtens, J. Bolk, and G. V. der Sande, Opt. Express 20, 28603 (2012).
[15] K. Thakulsukanant, B. Li, T. Memon, G. Mezosi, Z. Wang, M. Sorel, and S. Yu, J. Lightwave Technol. 27, 631 (2009).

[16] C. Born, M. Sorel, and S. Yu, IEEE J. Quantum Electron. 41, 261 (2005).

[17] I. Ermakov, S. Beri, M. Ashour, J. Danckaert, B. Docter, J. Bolk, X. Leijtens, and G. Verschaffelt, IEEE J. Quantum Electron. 48, 129 (2012).

[18] Z. Wang, G. Yuan, G. Verschaffelt, J. Danckaert, and S. Yu, IEEE Photon. Technol. Lett. 20, 1228 (2008).

[19] S. Balle, Phys. Rev. A 57, 1304 (1998).

[20] S. Balle, Opt. Lett. 27, 1923 (2002).

[21] J. Javaloyes and S. Balle, Phys. Rev. A 81, 062505 (2010).

[22] J. Javaloyes and S. Balle, IEEE J. Quantum Electron. 45, 431 (2009).

[23] S. Fürst, A. Pérez-Serrano, A. Scirè, M. Sorel, and S. Balle, Appl. Phys. Lett. 93, 251109 (2008).

[24] J. Javaloyes and S. Balle, IEEE J. Quantum Electron. 47, 1078 (2011).

[25] A. Pérez-Serrano, J. Javaloyes, and S. Balle, IEEE Photon. Technol. Lett. 25, 476 (2013).

[26] A. M. Yacomotti, L. Furfaro, X. Hachair, F. Pedaci, M. Giudici, J. R. Tredicce, J. Javaloyes, S. Balle, E. A. Viktorov, and P. Mandel, Phys. Rev. A 69, 053816 (2004).

[27] M. Ahmed and M. Yamada, IEEE J. Quantum Electron. 38, 682 (2002).

[28] A. Pérez-Serrano, J. Javaloyes, and S. Balle, Opt. Express 19, 3284 (2011).

[29] A. P. Bogatov, P. G. Eliseev, and B. N. Sverdlov, IEEE J. Quantum Electron. 11, 510 (1975).

[30] L. M. Narducci and N. B. Abraham, Laser Physics and Laser Instabilities (World Scientific Publishing, Singapore, 1988).

[31] J. Javaloyes, M. Perrin, G. L. Lippi, and A. Politi, Phys. Rev. A 70, 023405 (2004).

[32] J. Javaloyes, M. Perrin, and A. Politi, Phys. Rev. E 78, 011108 (2008).

[33] G. L. Gattobigio, F. Michaud, J. Javaloyes, J. W. R. Tabosa, and R. Kaiser, Phys. Rev. A 74, 043407 (2006). 
[34] J. Javaloyes and S. Balle, IEEE J. Quantum Electron. 48, 1519 (2012).

[35] J. Javaloyes and S. Balle, Opt. Express 20, 8496 (2012).

[36] J. Javaloyes and S. Balle, computer code FREETWM, http://onl.uib.es/es/Softwares/Freetwm/.

[37] A. Pérez-Serrano, J. Javaloyes, and S. Balle, Phys. Rev. A 81, 043817 (2010).

[38] O. Conradi, S. Helfert, and R. Pregla, IEEE J. Quantum Electron. 37, 928 (2001).

[39] E. Doedel, A. R. Champneys, T. F. Fairgrieve, Y. A. Kuznetsov, B. Sand-stede, and X. Wang, computer code AUTO, http://indy.cs.concordia.ca/auto/.

[40] K. Engelborghs, T. Luzyanina, G. Samaey, D. Roose, and K. Verheyden, computer code DDE-BIFTOOL, http://twr.cs. kuleuven.be/research/software/delay/ddebiftool.shtml.
[41] R. LeVeque, Finite Difference Methods for Ordinary and Partial Differential Equations: Steady-State and Time-Dependent Problems (Society for Industrial and Applied Mathematics, Philadelphia, 2007).

[42] W. Press, Numerical Recipes: The Art of Scientific Computing (Cambridge University Press, Cambridge, 2007).

[43] H. Risken and K. Nummedal, J. Appl. Phys. 39, 4662 (1968).

[44] L. M. Narducci, J. R. Tredicce, L. A. Lugiato, N. B. Abraham, and D. K. Bandy, Phys. Rev. A 33, 1842 (1986).

[45] L. A. Lugiato, L. M. Narducci, and M. F. Squicciarini, Phys. Rev. A 34, 3101 (1986).

[46] L. Gelens, G. Van der Sande, S. Beri, and J. Danckaert, Phys. Rev. E 79, 016213 (2009).

[47] P. Mandel and G. P. Agrawal, Opt. Commun. 42, 269 (1982).

[48] M. Sargent, III, Phys. Rev. A 48, 717 (1993). 